\title{
Production of conductive bacterial cellulose-polyaniline membranes in the presence of metal salts
}

Textile Research Journa $0(00) 1-10$

(C) The Author(s) 2019 Article reuse guidelines: sagepub.com/journals-permissions DOI: $10.1177 / 0040517519893717$ journals.sagepub.com/home/trj

SAGE

\author{
Hyunjin Kim', Ji Eun Song ${ }^{2}$, Carla Silva ${ }^{3}$ and Hye Rim Kim' ${ }^{\text {(D) }}$
}

\begin{abstract}
This study presents a cost-effective method of enhancing the electrical conductivity and washing durability of bacterial cellulose (BC)-polyaniline (PANI) membrane by the addition of metal salt. In this study, two types of metal salts were tested: copper (II) sulfate and iron (II) sulfate. The optimal condition to produce BC-PANI-metal salt membranes was $0.05 \%(\mathrm{w} / \mathrm{v})$ of copper (II) sulfate (copper salt). X-ray diffraction analysis showed that the crystallinity of BC-PANI increased after adding copper salt. According to the increased degree of crystallinity, the polymer chain structure of BC-PANI-copper salt (BC-PANI-Cu) was more organized than that of BC-PANI, as confirmed by scanning electron microscopy. In addition, this ordered structure of BC-PANI-Cu indicated enhanced electrical conductivity. Moreover, the addition of copper salt improved the electrical conductivity of BC-PANI to a level about 3.8 times higher than that of BC-PANI produced without metal salt, and it retained about $40 \%$ of its original electrical conductivity after three washing cycles. From the results, the addition of copper salt improved both the electrical conductivity and washing durability of the BC-PANI membrane.
\end{abstract}

\section{Keywords}

bacterial cellulose, polyaniline, metal salt, copper salt, electrical conductivity, washing durability

Conductive textiles are electrically functionalized materials manufactured by embroidering, knitting, weaving, or coating chemically synthesized polymers onto the textile structure. ${ }^{1,2}$ Among these, chemical synthesis and conductive polymer coating are the methods that are most widely used to produce conductive textiles because: (i) conductive materials can be simply fabricated without weaving or knitting, ${ }^{3,4}$ (ii) the electrical conductivity of conductive materials can be adjusted by varying the type of dopant acids involved in the polymerization process, ${ }^{5}$ and (iii) the mechanical properties of textile materials are not affected by chemical treatment. ${ }^{6}$ Conductive polymers exhibit extended $\pi$-electron delocalization along their conjugate backbone consisting of alternating single and double bonds, ${ }^{7,8}$ and hence they are lightweight and corrosion-resistant. ${ }^{9}$ Among these polymers, polyaniline (PANI) is easy to synthesize, cost-effective due to the low cost of monomer, and it shows good electrical conductivity and high chemical stability. ${ }^{10,11}$

Bacterial cellulose (BC) is a pure cellulose material produced by Acetobacter or Gluconacetobacter bacteria $^{12}$ and possesses excellent flexibility, high crystallinity, high porosity, three-dimensional nanostructure, good mechanical properties, and high waterholding capacity. ${ }^{13-15}$ Due to these characteristics, conductive polymers can be effectively polymerized inside the $\mathrm{BC}$ nanostructure. Hence, conductive BC membranes were produced by incorporating PANI

\footnotetext{
'Department of Clothing and Textiles, Sookmyung Women's University, Seoul, Korea

${ }^{2}$ Human Convergence Technology Group, Korea Institute of Industrial Technology, Ansan, Korea

${ }^{3}$ Center of Biological Engineering, University of Minho, Braga, Portugal

Corresponding authors:

Ji Eun Song, Human Convergence Technology Group, Korea Institute of Technology, Ansan 15588, Korea.

Email: mplala@kitech.re.kr

Hye Rim Kim, Department of Clothing and Textiles, Sookmyung Women's University, Cheongpa-ro 47-gil 100 (Cheongpa-dong 2(i)-ga), Yongsan-gu, Seoul 04310, Korea.

Email: khyerim@sookmyung.ac.kr
} 
into BC with an in-situ synthesis method and the BC polymerized with PANI was named BC-PANI. Several studies have reported the production of conductive BCPANI membranes. However, the electrical conductivity of BC-PANI membranes produced in previous studies is $1.61 \times 10^{-4} \sim 5.2 \times 10^{-3} \mathrm{~S} / \mathrm{cm}$, which is not high enough to be used as a conductive textile. ${ }^{16,17} \mathrm{~A}$ conductive textile requires good flexibility and durability in order to be worn. It must also maintain its electrical conductivity even after washing; however, there is no study to evaluate the washing durability of BC-PANI. Thus, simple and cost-effective methods to improve the electrical conductivity and to maintain the washing durability of BC-PANI are required.

This study aims to improve the electrical conductivity of BC-PANI and to impart washing durability to it by employing additives. The electrical conductivity of PANI can be enhanced by using additives such as carbon nanotubes, ${ }^{18}$ graphene, ${ }^{19}$ and metal salts. ${ }^{20}$ In particular, metal salts are inexpensive and easy to handle during the polymerization of PANI. Moreover, using metal salts improves the mechanical properties of PANI. ${ }^{21,22}$ Despite these advantages, few studies have been conducted to synthesize PANI with metal salts. ${ }^{23-25}$ Thus, in this study, two metal salts - copper (II) sulfate and iron (II) sulfate - were used. The conductive BC-PANI produced with the metal salts was denominated as BC-PANI-metal salt. Two types of BC-PANI-metal salt membranes were produced and the effects of the metal salts on the electrical conductivity of BC-PANI were investigated. The polymerization conditions for the BC-PANI-metal salt membranes were optimized by measuring their electrical conductivity using the four-point probe method. The polymerization of PANI was assessed by ultraviolet-visible (UV-Vis) spectroscopy and matrix-assisted laser desorption/ionization-time of flight (MALDI-TOF) analyses. The chemical and physical characteristics of the BCPANI-metal salt membrane produced under the optimized conditions were evaluated by Fourier-transform infrared (FT-IR) spectroscopy, X-ray diffraction (XRD), field-emission scanning electron microscopy (FE-SEM), and washing durability analysis.

\section{Experimental details}

\section{Materials}

Yeast extract and peptone were purchased from BD Biosciences (San Jose, CA, USA). Glucose, hydrogen peroxide $(34.5 \%)$, sodium hydroxide, and acetic acid were obtained from Duksan Pure Chemical Co., Ltd. (Seoul, Korea). Dodecylbenzene sulfonic acid (DBSA) was obtained from Sigma-Aldrich (St. Louis, MO, USA). Aniline was obtained from Junsei Chemical
Co., Ltd. (Tokyo, Japan). Ammonium persulfate (APS) was obtained from Kanto Chemical Co., Inc. (Tokyo, Japan). Ethyl alcohol (99.9\%) and iron (II) sulfate $\left(\mathrm{FeSO}_{4} \cdot 7 \mathrm{H}_{2} \mathrm{O}\right)$ were obtained from Duksan Pure Chemical Co., Ltd. (Seoul, Korea), whereas copper (II) sulfate $\left(\mathrm{CuSO}_{4} \cdot 5 \mathrm{H}_{2} \mathrm{O}\right)$ was purchased from Shinyo Pure Chemicals Co., Ltd. (Osaka, Japan). All the chemical reagents were used as-received without further purification.

\section{Methods}

The BC-PANI-metal salt membranes were prepared using an in-situ synthesis method. BC was produced according to the procedure reported by Han et al. ${ }^{26}$ and was then pre-treated by washing, bleaching, and swelling according to previously reported procedures. $^{26,27}$ Figure 1 illustrates the synthesis of the BCPANI-metal salt membranes. First, pre-treated BC $(2 \mathrm{~cm} \times 2 \mathrm{~cm})$, aniline $(2.5 \mathrm{mM})$, and the two types of metal salts (copper (II) sulfate and iron (II) sulfate) were added to a DBSA solution $(2.5 \mathrm{mM})$ with a $\mathrm{pH}$ of 2.5. The resulting solution was magnetically stirred for $30 \mathrm{~min}$. Next, an APS solution $(2.5 \mathrm{mM})$ was added to the reaction mixture to initiate the polymerization process. The resulting solution was polymerized for $24 \mathrm{~h}$ at $20^{\circ} \mathrm{C}$. Finally, BC-PANI-metal salt membranes were obtained and dried in a drying oven for $4 \mathrm{~h}$ at $20^{\circ} \mathrm{C}$.

\section{Characterization}

Electrical conductivity. Electrical conductivity measurements were carried out to optimize the polymerization conditions of BC-PANI-metal salt. The electrical resistivity of the BC-PANI-metal salt membranes was measured using the four-point probe method (CMT-SR1000N, Chang Min Tech Co., Ltd., Korea). The electrical resistivity of each sample was measured at five different points and the average value of these measurements was reported. The electrical conductivity of the samples was then calculated using Equation (1):

$$
\sigma=\frac{1}{(R \times d)}
$$

where $\sigma, R$, and $d$ are the electrical conductivity $(\mathrm{S} / \mathrm{cm})$, electrical resistivity $(\Omega \cdot \mathrm{cm})$, and thickness $(\mathrm{cm})$ of the samples, respectively.

UV-Vis spectroscopy. To confirm the polymerization of PANI, the UV-Vis spectra of the BC-PANI-metal salt polymerization solutions were obtained using a 96quartz microplate reader (SynergyMx, Shimadzu, Japan). The UV-Vis spectra were recorded over the wavelength range of $300-800 \mathrm{~nm}$. 


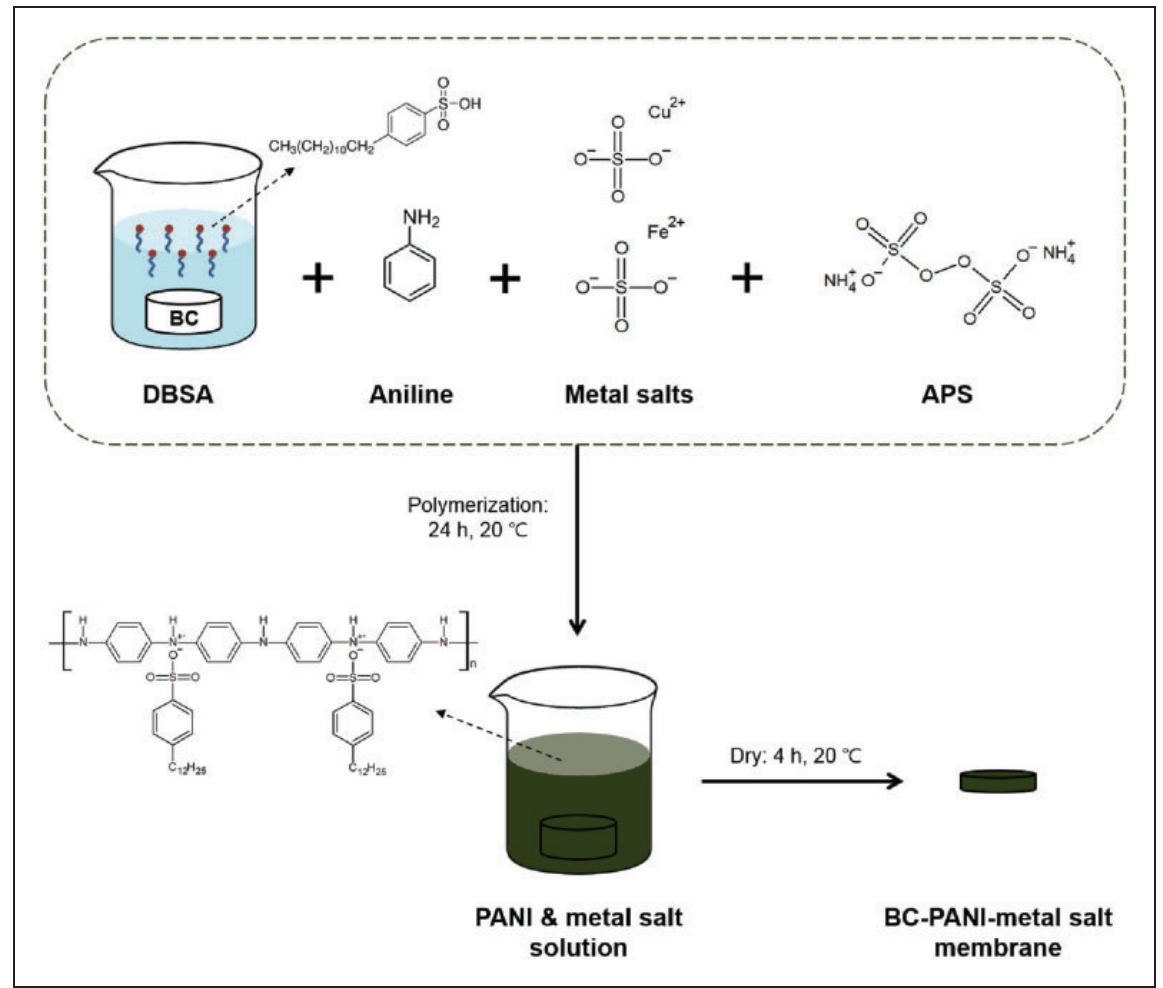

Figure I. Schematic illustration of the in-situ synthesis of the BC-PANI-metal salt membranes.

MALDI-TOF mass spectrometry. The degree of polymerization (DP) of PANI was analyzed by MALDI-TOF mass spectrometry using $\alpha$-cyano-4-hydroxycinnamic acid (CHCA) as the matrix. The mass spectra of the samples were obtained using an Ultra-flex MALDITOF mass spectrophotometer (Bruker Daltonics $\mathrm{GmbH}$, Bremen, Germany) equipped with a $337 \mathrm{~nm}$ nitrogen laser in the linear positive mode. All the samples were dissolved in a TA30 $(30 \%$ acetonitrile/ $70 \%$ trifluoracetic acid) solution, and to the resulting solutions, a saturated solution of CHCA was added. A sample $(5 \mu \mathrm{L})$ of the resulting solution was then dropped on a ground steel plate (Bruker part n. 209519) and dried.

FT-IR spectroscopy. A Nicolet IS50 (Thermo Fisher Scientific, Waltham, MA, USA) FT-IR spectrophotometer was used to examine the chemical structure of the BC-PANI-metal salt membrane prepared under the optimized conditions. The FT-IR spectra of the samples were recorded over the wavenumber range of $650-4,000 \mathrm{~cm}^{-1}$ at a resolution of $4 \mathrm{~cm}^{-1}$. For each spectrum, baseline normalization was carried out using OMNIC software (Thermo Fisher Scientific, Waltham, MA, USA).

XRD. The crystalline structure of the BC-PANI-metal salt membrane obtained under the optimized conditions was examined using a D8 Advance diffractometer (Bruker AXS Inc., Karlsruhe, Germany) over the $2 \theta$ range of $0^{\circ}-40^{\circ}$. XRD measurements were carried out with a $\mathrm{Cu}-\mathrm{K}_{\alpha}$ radiation source $(\lambda=1.5406 \mathrm{~nm})$ at $40 \mathrm{kV}$ and $40 \mathrm{~mA}$.

Surface morphology. The surface morphology of the BC-PANI-metal salt membrane prepared under the optimized conditions was examined by FE-SEM (JSM-7600F, JEOL Ltd., Tokyo, Japan). All the samples were sputter-coated with platinum using a magnetron sputter coater (108auto, Cressington Scientific Instruments, Watford, UK).

Washing durability. Washing durability tests were carried out in three sequential washing steps. Each step was performed in a shaking water bath (BS-21, JEIO TECH Co., Daejeon, Korea) at a rotating speed of $110 \mathrm{rpm}$ at $25^{\circ} \mathrm{C}$ for $120 \mathrm{~min}$. After each step, the samples were dried for $1 \mathrm{~h}$ at $25^{\circ} \mathrm{C}$. The electrical conductivity of each of the samples was evaluated after each washing step and the relative change in the electrical conductivity was calculated using Equation (2):

$$
\frac{\Delta R}{R_{0}}=\frac{\left(R-R_{0}\right)}{R_{0}}
$$


Table I. Electrical conductivity of BC-PANI and BC-PANI-metal salt membranes (polymerization conditions: $\mathrm{pH}$ of the DBSA solution $=2.5$, DBSA:aniline:APS $=1: 1: 1,20^{\circ} \mathrm{C}, 24 \mathrm{~h}$ )

\begin{tabular}{llllll}
\hline \multirow{5}{*}{ Sample } & \multicolumn{3}{l}{ Electrical conductivity $(\mathrm{S} / \mathrm{cm})$} & & \\
\cline { 2 - 6 } & Metal salt & $0.05 \%(\mathrm{w} / \mathrm{v})$ & $0.1 \%(\mathrm{w} / \mathrm{v})$ & $0.5 \%(\mathrm{w} / \mathrm{v})$ & $1.0 \%(\mathrm{w} / \mathrm{v})$ \\
\hline BC-PANI & - & $1.99 \times 10^{-2}$ & & & \\
BC-PANI-Cu & Copper & $7.54 \times 10^{-2}$ & $2.54 \times 10^{-5}$ & $5.66 \times 10^{-7}$ & $1.49 \times 10^{-6}$ \\
BC-PANI-Fe & Iron & $7.47 \times 10^{-6}$ & $6.97 \times 10^{-7}$ & $9.69 \times 10^{-7}$ & $9.8 \mathrm{I} \times 10^{-8}$ \\
\hline
\end{tabular}

\section{Results and discussion}

PANI was polymerized in-situ with BC to prepare conductive BC-PANI membranes. Copper (II) sulfate and iron (II) sulfate were added to improve the electrical conductivity of BC-PANI. The BC-PANI samples polymerized with copper (II) sulfate and iron (II) sulfate were labeled as BC-PANI-Cu and BC-PANI-Fe, respectively. The electrical conductivity and degrees of polymerization of the two membranes were evaluated and compared.

\section{Electrical conductivity}

The electrical conductivity of the BC-PANI-metal salt membranes was determined using the four-point probe method. As can be observed from Table 1, the BCPANI-Cu membrane polymerized using 0.05\% (w/v) copper (II) sulfate showed the highest electrical conductivity of $7.54 \times 10^{-2} \mathrm{~S} / \mathrm{cm}$ which was about 3.8 times higher than that of BC-PANI. It also explains the improvement in the electrical conductivity of BC-PANI more than 14.5 times compared with that of BC-PANI from previous studies. ${ }^{16,17}$ With an increase in the copper salt concentration, the electrical conductivity of the BCPANI-Cu membrane decreased. This can be attributed to the over-oxidation of PANI because copper (II) sulfate acts as an oxidizing agent. ${ }^{28}$ As PANI is polymerized through the oxidation of aniline, the amount of oxidizing agent affects the electrical conductivity of PANI. ${ }^{29}$ In the case of the BC-PANI-Fe membrane, the iron salt acts as the reducing agent. ${ }^{30}$ The electrical conductivity of the BC-PANI-Fe membrane decreased with an increase in the iron salt concentration. On the basis of these results, copper (II) sulfate concentration of $0.05 \%(\mathrm{w} / \mathrm{v})$ was found to be optimum for producing BC-PANI-metal salt membranes.

\section{Polymerization of PANI}

Figure 2 shows the UV-Vis spectra of the polymerization solutions of BC-PANI, BC-PANI-Cu, and BCPANI-Fe with the highest electrical conductivity. The BC-PANI and BC-PANI-Cu polymerization solutions showed similar characteristic bands with three

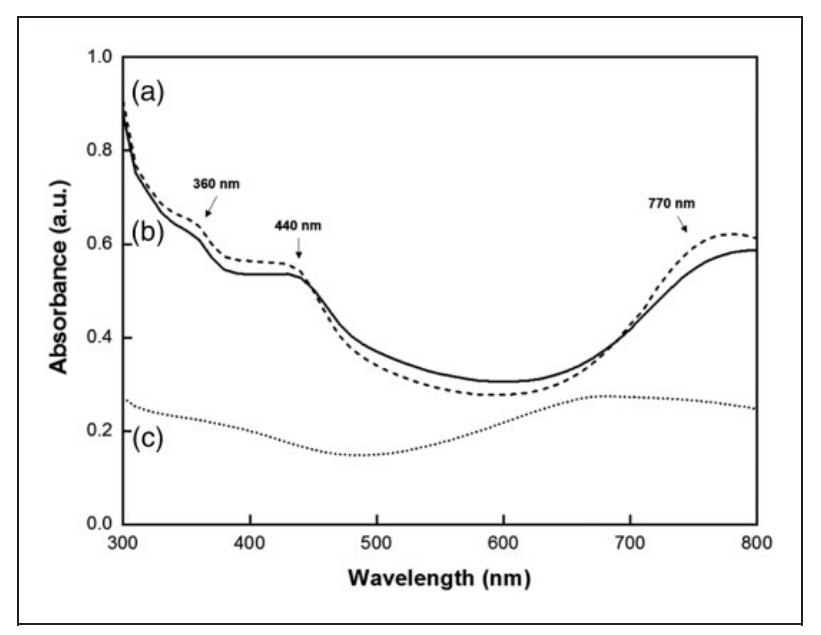

Figure 2. UV-Vis spectra of the polymerization solutions of (a) BC-PANI, (b) BC-PANI-Cu, and (c) BC-PANI-Fe (polymerization conditions: $0.05 \%(\mathrm{w} / \mathrm{v})$ of metal salts, $\mathrm{pH}$ of the DBSA solution $=2.5$, DBSA:aniline:APS $=\mathrm{I}: \mathrm{I}: \mathrm{I}, 20^{\circ} \mathrm{C}, 24 \mathrm{~h}$ ).

noticeable peaks at around 360, 440, and $770 \mathrm{~nm}$. These peaks indicate that PANI exists in the conductive emeraldine salt state. ${ }^{31}$ In particular, the peak at around $360 \mathrm{~nm}$ corresponds to the $\pi-\pi *$ electronic transition along the PANI backbone, while that at $440 \mathrm{~nm}$ corresponds to the exciton transition caused by the doping of PANI. ${ }^{32}$ The absorption bands at around $600-700 \mathrm{~nm}$ and $700-800 \mathrm{~nm}$ can be attributed to the formation of polarons. ${ }^{33}$ However, the polymerization solution of BC-PANI-Fe showed no peak corresponding to PANI. This indicates that the addition of the iron salt inhibited the polymerization of PANI.

The MALDI-TOF mass spectra of the membranes were examined to determine the degrees of polymerization of PANI. Figure 3 shows the MALDI-TOF mass spectra of BC-PANI, BC-PANI-Cu, and BC-PANI-Fe. The mass spectrum of BC-PANI showed peaks at 98, $189,270,372,465$, and $557 \mathrm{~m} / z$ (mass-to-charge ratio). Since the $m / z$ value of the monomer unit of PANI $\left(\mathrm{C}_{6} \mathrm{NH}_{5}\right)$ is $91,{ }^{34-36}$ the peaks at $98,189,270$, and $372 \mathrm{~m} / \mathrm{z}$ can be attributed to aniline monomers, dimers, trimers, and tetramers, respectively. The peak 


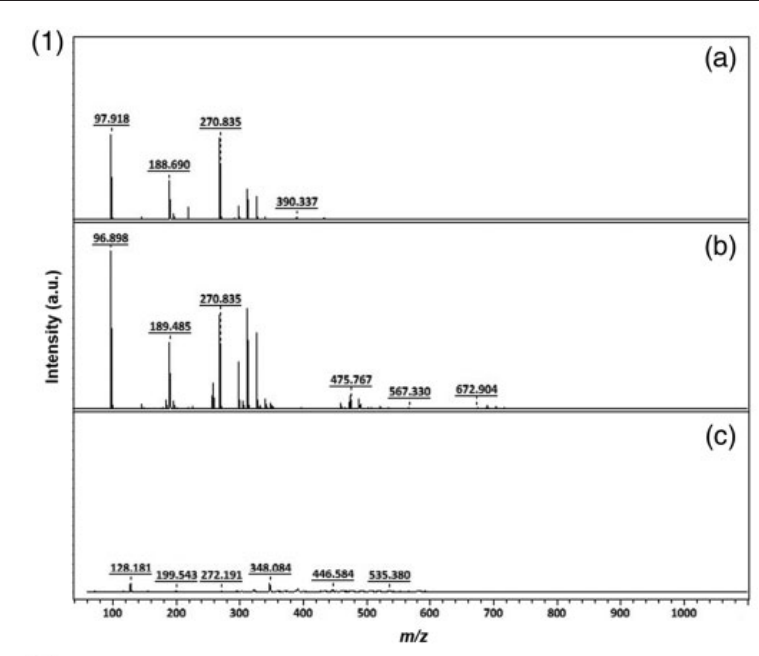

(2)

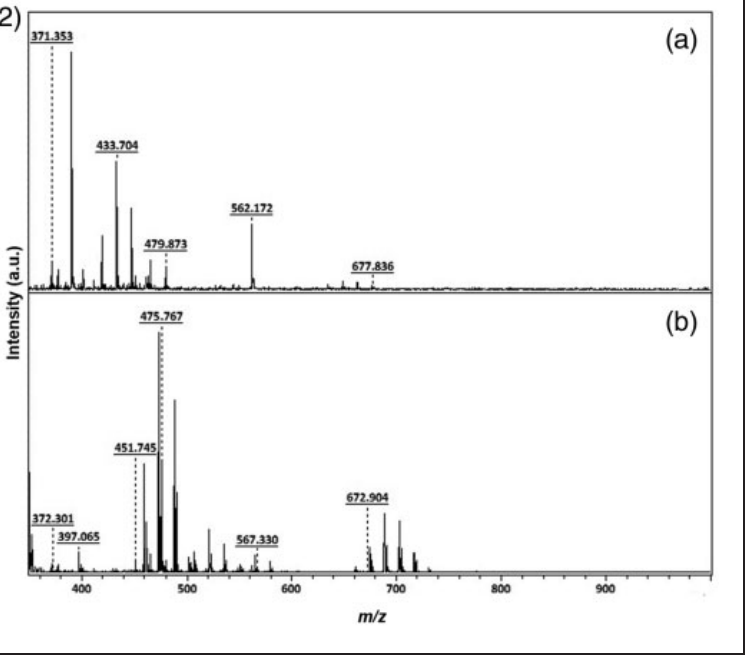

Figure 3. (I) MALDI-TOF mass spectra of (a) BC-PANI, (b) BC-PANI-Cu, and (c) BC-PANI-Fe. (2) Enlarged view of the MALDI-TOF mass spectra of (a) BC-PANI and (b) BC-PANI-Cu over the $350-1,000 \mathrm{~m} / \mathrm{z}$ range (polymerization conditions: $0.05 \%$ $(\mathrm{w} / \mathrm{v})$ of metal salts, $\mathrm{pH}$ of the DBSA solution $=2.5$,

DBSA:aniline:APS $=1: 1: 1,20^{\circ} \mathrm{C}, 24 \mathrm{~h}$ ).

at $372 \mathrm{~m} / z$ (Figure 3) corresponds to PANI, since the smallest repeat unit of the PANI emeraldine state is the aniline tetramer. ${ }^{37}$ The peak corresponding to the aniline tetramer was also observed in the mass spectrum of BC-PANI-Cu, confirming the polymerization of PANI. In addition, as can be observed from Table 2, BCPANI-Cu exhibited the highest DP of PANI. In the case of BC-PANI-Fe, the polymerization of PANI could not be confirmed because the peak intensities were very low and the peak corresponding to the monomer was not detected. This can be attributed to the use of the iron salt as the reducing agent in this case. ${ }^{30}$ Thus, the MALDI-TOF results showed that the addition of the copper salt promoted the polymerization of PANI.
Table 2. DP, molecular weight, and polydispersity index of BCPANI and BC-PANI-Cu (polymerization conditions: $0.05 \%(\mathrm{w} / \mathrm{v})$ of metal salts, $\mathrm{pH}$ of the DBSA solution $=2.5$, DBSA:aniline:APS $=1: 1: 1,20^{\circ} \mathrm{C}, 24 \mathrm{~h}$ )

\begin{tabular}{llll}
\hline & $D_{\max }$ & $\mathrm{M}_{\mathrm{w}} *$ & $\mathrm{PDI}^{* *}$ \\
\hline BC-PANI & 6.11 & 260.11 & 1.17 \\
BC-PANI-Cu & 7.95 & 308.84 & 1.22
\end{tabular}

Notes: *Molecular weight. **Polydispersity index

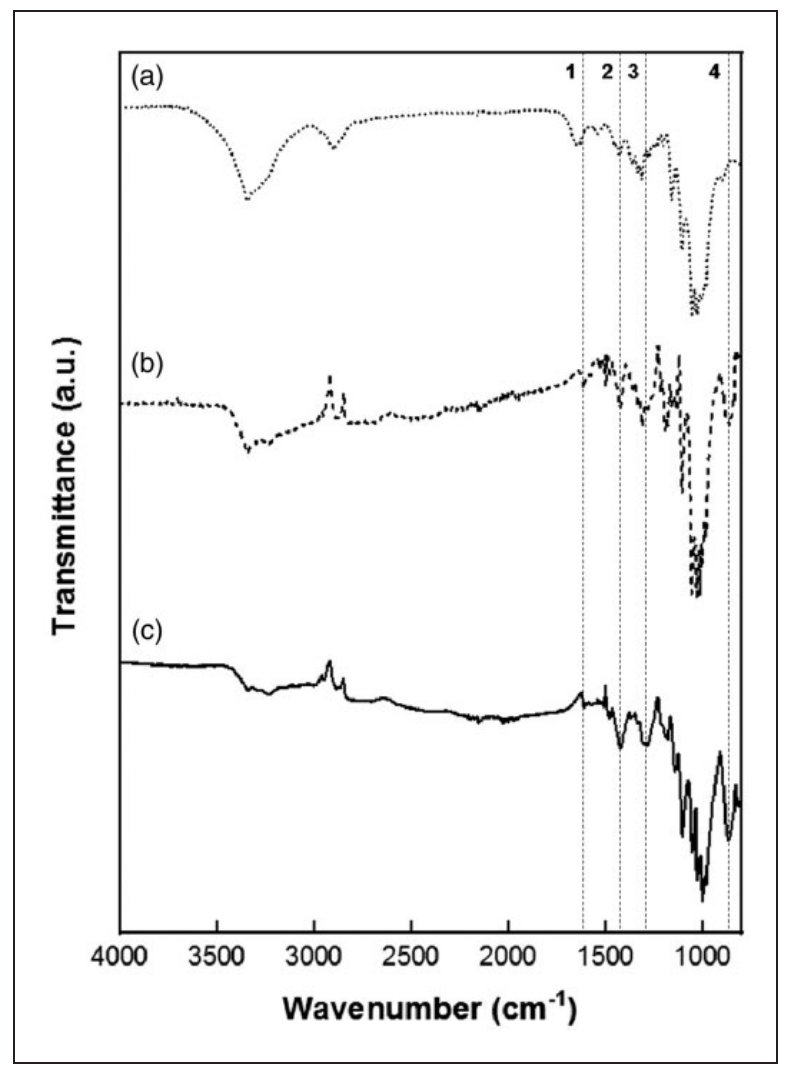

Figure 4. FT-IR spectra of (a) untreated BC, (b) BC-PANI, and (c) BC-PANI-Cu (polymerization conditions: $0.05 \%(w / v)$ of copper (II) sulfate, $\mathrm{pH}$ of the DBSA solution $=2.5$, DBSA:aniline:APS $=\mathrm{I}: \mathrm{l}: \mathrm{l}, 20^{\circ} \mathrm{C}, 24 \mathrm{~h}$ ).

\section{Characterization of BC-PANI-Cu}

The chemical structure of BC-PANI-Cu was examined by FT-IR spectroscopy. As can be observed from Figure 4 , in the case of BC-PANI and BC-PANI-Cu, the intensities of the peaks at $3550-3230 \mathrm{~cm}^{-1}$ corresponding to the $-\mathrm{OH}$ vibration of hydrogen bonds in cellulose decreased. ${ }^{38}$ This suggests that the $-\mathrm{OH}$ functional groups in $\mathrm{BC}$ reacted with PANI during the polymerization process. ${ }^{39}$ BC-PANI and BC-PANI-Cu showed the characteristic peaks of PANI (Table 3). 
Table 3. Characteristic peaks of PANI in the FT-IR spectra of BC-PANI and BC-PANI-Cu (polymerization conditions: $0.05 \%$ (w/v) of copper (II) sulfate, $\mathrm{pH}$ of the DBSA solution $=2.5$, DBSA:aniline:APS $=\mathrm{I}: \mathrm{I}: \mathrm{I}, 20^{\circ} \mathrm{C}, 24 \mathrm{~h}$ )

\begin{tabular}{llll}
\hline & \multicolumn{2}{l}{ Wavenumber $\left(\mathrm{cm}^{-1}\right)$} & \\
\cline { 2 - 3 } Peak number & BC-PANI & BC-PANI-Cu & Peak assignment \\
\hline $\mathrm{I}$ & 1612 & $161 \mathrm{I}$ & $\mathrm{C}=\mathrm{C}$ stretching vibration of quinoid ring \\
2 & 1425 & 1422 & $\mathrm{C}=\mathrm{C}$ stretching vibration of benzenoid ring $^{43,44}$ \\
3 & 1313 & 1290 & C-N stretching vibration of secondary aromatic amine \\
4 & 861 & 864 & C-H out-of-plane bending vibration of I,4-disubstituted aromatic benzene ring \\
\hline
\end{tabular}

The peaks corresponding to PANI shifted slightly in the case of BC-PANI-Cu because of the chemical reaction between the copper salt and the reaction sites (amine and imine groups) of PANI. ${ }^{40}$ The absorption peaks at $1612,1611,1425$, and $1422 \mathrm{~cm}^{-1}$ correspond to the amine and imine groups in BC-PANI and BCPANI-Cu. ${ }^{41-44}$ The peaks at 1313 and $1290 \mathrm{~cm}^{-1}$ correspond to the protonated state of PANI, ${ }^{45,46}$ while the peaks at 861 and $864 \mathrm{~cm}^{-1}$ can be attributed to the head-to-tail coupling of the aniline monomers. ${ }^{47,48}$ Thus, the FT-IR analysis confirmed the presence of PANI in the BC-PANI-Cu membrane.

Figure 5 shows the XRD patterns of untreated BC, BC-PANI, and BC-PANI-Cu membranes. All the samples showed diffraction peaks at $2 \theta=14.7^{\circ}$ and $22.8^{\circ}$, indicating the presence of the cellulosic structure. ${ }^{15}$ This suggests that the cellulose structure of $\mathrm{BC}$ was retained when the polymerization was carried out in the presence of copper. The diffraction peak at $2 \theta=25^{\circ}$ in the XRD pattern of BC-PANI confirms the formation of PANI. ${ }^{49}$ In the case of BC-PANI-Cu, the diffraction peak of PANI shifted slightly to $24.2^{\circ} .^{50}$ This can be attributed to the effect of the copper salt on the polymerization of PANI. The narrow peak at $2 \theta=18.7^{\circ}$ in the diffraction pattern of BC-PANI-Cu can be attributed to the presence of copper. ${ }^{51}$ As in Table 4 , the degree of crystallinity of BC-PANI was decreased. It was then increased after adding copper salt. This can be attributed to the reaction of the $-\mathrm{OH}$ functional groups of $\mathrm{BC}$ with the copper salt, leading to an ordered arrangement of the polymer chains. ${ }^{52}$ This is consistent with the results reported previously that the structural ordering of $\mathrm{BC}$ improves its electrical conductivity. ${ }^{12,53}$ The XRD results showed that the copper salt improved the degree of crystallinity of the BC-PANI membrane.

The surface morphology of BC-PANI-Cu was examined by SEM (Figure 6). The thickness of the untreated BC (Figure 6(a)) fibers increased with their treatment with PANI. This is because the fibers bundled together with the polymerization of PANI. ${ }^{17,54}$ Moreover, the structure of BC-PANI-Cu (Figure 6(c)) was more

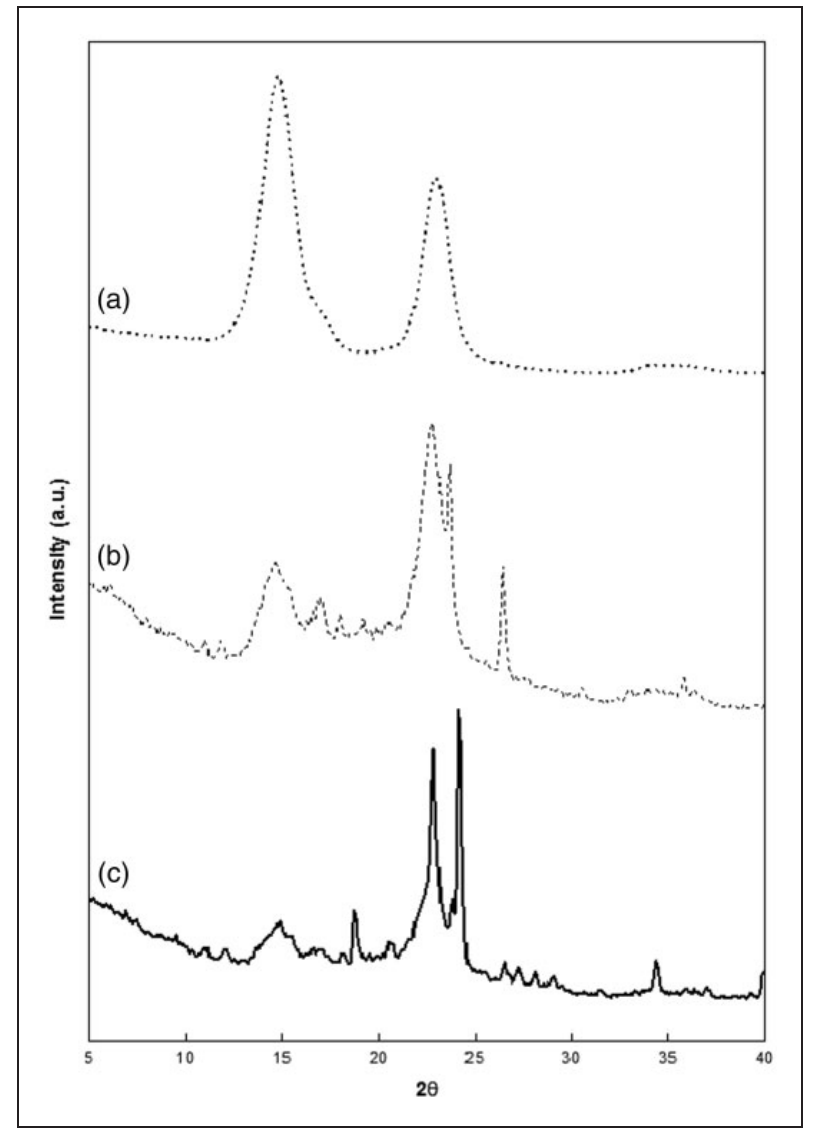

Figure 5. XRD patterns of (a) untreated $B C$, (b) BC-PANI, and (c) BC-PANI-Cu (polymerization conditions: $0.05 \%(\mathrm{w} / \mathrm{v})$ of copper (II) sulfate, $\mathrm{pH}$ of the DBSA solution $=2.5$, DBSA:aniline:APS $=\mathrm{I}: \mathrm{I}: \mathrm{l}, 20^{\circ} \mathrm{C}, 24 \mathrm{~h}$ ).

Table 4. Degrees of crystallinity of untreated BC, BC-PANI, and BC-PANI-Cu (polymerization conditions: $0.05 \%(\mathrm{w} / \mathrm{v})$ of copper (II) sulfate, $\mathrm{pH}$ of the DBSA solution $=2.5$, DBSA:aniline:APS $=1: 1: 1,20^{\circ} \mathrm{C}, 24 \mathrm{~h}$ )

\begin{tabular}{ll}
\hline Sample & Crystallinity (\%) \\
\hline Untreated BC & 77.4 \\
BC-PANI & 65.0 \\
BC-PANI-Cu & 72.8 \\
\hline
\end{tabular}




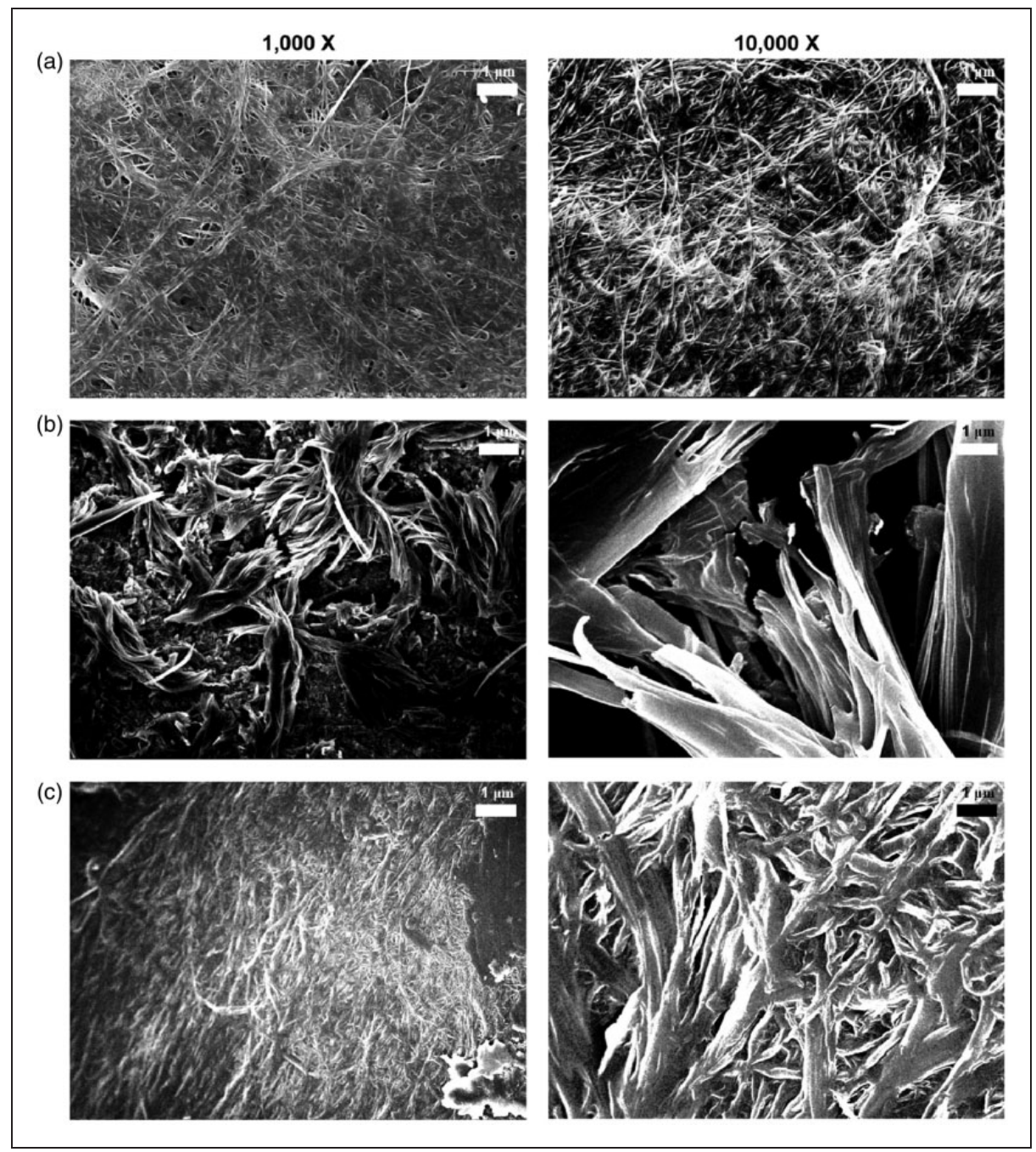

Figure 6. SEM images of (a) untreated BC, (b) BC-PANI, and (c) BC-PANI-Cu at I,000 and 10,000 X magnification with a scale bar of I $\mu \mathrm{m}$ (polymerization conditions: $0.05 \%(\mathrm{w} / \mathrm{v})$ of copper (II) sulfate, $\mathrm{pH}$ of the DBSA solution $=2.5$, DBSA:aniline:APS $=1: \mathrm{I}: \mathrm{I}, 20^{\circ} \mathrm{C}, 24 \mathrm{~h}$ ).

organized than that of BC-PANI (Figure 6(b)). This can be attributed to the increased degree of crystallinity of BC-PANI with the addition of the copper salt, thus facilitating the polymer chain arrangement. ${ }^{52}$

Washing durability tests were carried out to examine the changes in the electrical conductivity of BC-PANI and BC-PANI-Cu after washing. As can be observed from Figure 7, the electrical conductivity of BC-PANI$\mathrm{Cu}$ decreased by $41 \%$, while that of BC-PANI decreased by $83 \%$ after the first washing. After three washing steps, BC-PANI-Cu retained its original electrical conductivity over $40 \%$ while that of BC-PANI was maintained by $1.4 \%$. This indicates that the copper salt strengthened the bond between the $\mathrm{BC}$ fibers and PANI. ${ }^{55}$ Thus, it was confirmed that the addition of the copper salt increased the washing durability of the BC-PANI membrane.

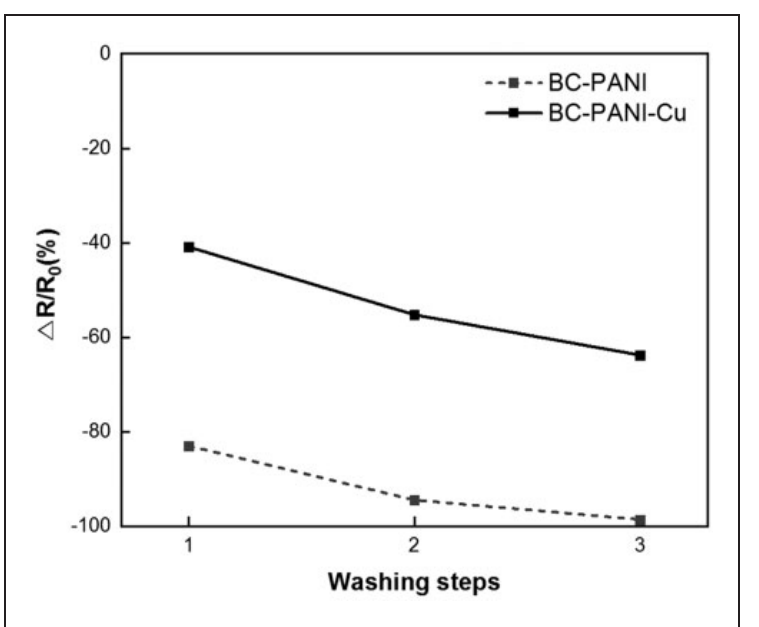

Figure 7. Changes in the relative electrical conductivity of BCPANI and BC-PANI-Cu after different washing steps. 


\section{Conclusions}

The aim of this work was to improve the electrical conductivity and to impart washing durability to BC-PANI by employing inexpensive additives. To achieve this, a conductive BC-PANI was prepared by incorporating PANI polymer in $\mathrm{BC}$ membranes by the in-situ synthesis method. The polymerization conditions of PANI were evaluated on the basis of the electrical conductivity of the BC-PANI-metal salt membranes. The addition of $0.05 \%(\mathrm{w} / \mathrm{v})$ of copper (II) sulfate was found to be optimum for the preparation of the BC-PANI-metal salt membranes. The electrical conductivity of BC-PANI-Cu was $7.54 \times 10^{-2} \mathrm{~S} / \mathrm{cm}$, which was 3.8 times greater than that of BC-PANI. It was also more than 14.5 times greater than that of BC-PANI from previous studies. The UV-Vis results showed that PANI existed in the emeraldine state in BC-PANI-Cu. The MALDI-TOF results explained that BC-PANI-Cu exhibited a higher degree of PANI polymerization than the other membranes, confirming that the addition of copper improved the polymerization degree of PANI. The BC-PANI-Cu membrane prepared under the optimum polymerization conditions was characterized by FT-IR, XRD, SEM, and washing durability tests. The FT-IR spectrum of BC-PANI-Cu showed characteristic peaks of PANI. The XRD results showed that the addition of copper improved the degree of crystallinity of the BC-PANI membrane. The SEM images of the membranes identified that BC-PANI-Cu exhibited a more ordered structural arrangement of $\mathrm{BC}$ fibers than BC-PANI. The washing durability of BC-PANI was improved by the addition of copper salt. After washing, the conductivity of BC-PANI with copper salt retained over $40 \%$ of the original electrical conductivity while that of BC-PANI was reduced to $1.4 \%$. This study provided a cost-effective approach for enhancing the electrical conductivity and washing durability of BC-PANI by employing copper salts.

\section{Declaration of conflicting interests}

The author(s) declared no potential conflicts of interest with respect to the research, authorship, and/or publication of this article.

\section{Funding}

The author(s) disclosed receipt of the following financial support for the research, authorship, and/or publication of this article: This work was supported by the National Research Foundation of Korea (NRF) grant funded by the Korean government (MSIT) (No. NRF-2019R1A2C1009217).

\section{ORCID iD}

Hye Rim Kim https://orcid.org/0000-0003-3456-8617

\section{References}

1. Stoppa M and Chiolerio A. Wearable electronics and smart textiles: a critical review. Sensors 2014; 14(7): 11957-11992.

2. Linz T, Von Krshiwoblozki M, Walter H, et al. Contacting electronics to fabric circuits with nonconductive adhesive bonding. J Text Inst 2012; 103(10): 1139-1150.

3. Knittel D and Schollmeyer E. Electrically high-conductive textiles. Synth Met 2009; 159(14): 1433-1437.

4. Ko J, Jee S, Lee J, et al. High durability conductive textile using MWCNT for motion sensing. Sens Actuators, A. 2018; 274: 50-56.

5. Kim S, Seong $\mathbf{J}$ and Oh K. Effect of dopant mixture on the conductivity and thermal stability of polyaniline/ nomex conductive fabric. J Appl Polym Sci 2002; 83(10): 2245-2254.

6. Šafářová V and Militký J. Multifunctional metal composite textile shields against electromagnetic radiation effect of various parameters on electromagnetic shielding effectiveness. Polym Compos 2015; 38(2): 309-323.

7. Baniasadi H, Ahmad Ramazani SA, Mashayekhan S, et al. Preparation of conductive polyaniline/graphene nanocomposites via in situ emulsion polymerization and product characterization. Synth Met 2014; 196: 199-205.

8. Molapo K, Ndangili P, Ajayi R, et al. Electronics of conjugated polymers (I): Polyaniline. Int J Electrochem Sci 2012; 7(12): 11859-11875.

9. Palaniappan S and John A. Polyaniline materials by emulsion polymerization pathway. Prog Polym Sci 2008; 33(7): 732-758.

10. Bhadra S, Khastgir D, Singha N, et al. Progress in preparation, processing and applications of polyaniline. Prog Polym Sci 2009; 34(8): 783-810.

11. Ćirić-Marjanović G. Recent advances in polyaniline research: Polymerization mechanisms, structural aspects, properties and applications. Synth Met 2013; 177: 1-47.

12. Lee B, Kim H and Yang H. Polymerization of aniline on bacterial cellulose and characterization of bacterial cellulose/polyaniline nanocomposite films. Curr Appl Phys 2012; 12(1): 75-80.

13. Torres F, Commeaux $\mathrm{S}$ and Troncoso $\mathrm{O}$. Biocompatibility of bacterial cellulose based biomaterials. J Funct Biomater 2012; 3(4): 864-878.

14. Lee H, Chung T, Kwon H, Kim H and Tze W. Fabrication and evaluation of bacterial cellulose-polyaniline composites by interfacial polymerization. Cellulose. 2012; 19(4): 1251-1258.

15. Wan Y, Li J, Yang Z, Ao H, Xiong L and Luo H. Simultaneously depositing polyaniline onto bacterial cellulose nanofibers and graphene nanosheets toward electrically conductive nanocomposites. Curr Appl Phys 2018; 18(8): 933-940.

16. Marins J, Soares B, Dahmouche K, et al. Structure and properties of conducting bacterial cellulose-polyaniline nanocomposites. Cellulose 2011; 18(5): 1285-1294.

17. Yue L, Xie Y, Zheng Y, et al. Sulfonated bacterial cellulose/polyaniline composite membrane for use as gel polymer electrolyte. Compos Sci Technol 2017; 145: 122-131. 
18. Oueiny C, Berlioz S and Perrin F. Carbon nanotubepolyaniline composites. Prog Polym Sci 2014; 39(4): 707-748.

19. Solonaru A and Grigoras M. Water-soluble polyaniline/ graphene composites as materials for energy storage applications. eXPRESS Polym Lett 2017; 11(2): 127-139.

20. Cirić-Marjanović G. Recent advances in polyaniline composites with metals, metalloids and nonmetals. Synth Met 2013; 170: 31-56.

21. Mallick K, Witcomb M and Scurrell M. Preparation and characterization of a conjugated polymer and copper nanoparticle composite material: A chemical synthesis route. Mater Sci Eng, B 2005; 123(2): 181-186.

22. Husain J, Pradeep P, Raghu N, et al. Synthesis, conductivity and sensitivity studies of polyaniline-iron oxide nanocomposites. Ferroelectrics 2016; 505(1): 229-235.

23. Kose T. Studies on transport properties of polyaniline transition metal salt composites. J Compos Mater 2011; 45(7): 831-837.

24. Virji S, Fowler J, Baker C, et al. Polyaniline nanofiber composites with metal salts: chemical sensors for hydrogen sulfide. Small 2005; 1(6): 624-627.

25. Kazemi S, Kiani M, Mohamadi R, et al. Metal-polyaniline nanofibre composite for supercapacitor applications. Bull Mater Sci 2014; 37(5): 1001-1006.

26. Han J, Shim E and Kim H. Effects of cultivation, washing, and bleaching conditions on bacterial cellulose fabric production. Text Res J 2018; 89(6): 1094-1104.

27. Song J, Su J, Loureiro A, et al. Ultrasound-assisted swelling of bacterial cellulose. Eng Life Sci 2017; 17(10): 1108-1117.

28. Mallick K, Witcomb MJ, Dinsmore A et al. Polymerization of aniline by cupric sulfate: A facile synthetic route for producing polyaniline. J Polym Res 2006; 13(5): 397-401.

29. Shah A, Kamran M, Bilal S, et al. Cost effective chemical oxidative synthesis of soluble and electroactive polyaniline salt and its application as anticorrosive agent for steel. Materials 2019; 12(9): 1527.

30. Mončeková M, Novotný R, Koplík J, et al. Hexavalent chromium reduction by ferrous sulphate heptahydrate addition into the portland clinker. Procedia Eng 2016; 151: 73-79.

31. Razak S, Ahmad A and Zein S. Polymerisation of protonic polyaniline/multi-walled carbon nanotubes-manganese dioxide nanocomposites. J Phys Sci 2009; 20(1): 27-34.

32. Bongiovanni Abel S, Molina M, Rivarola C, et al. Smart polyaniline nanoparticles with thermal and photothermal sensitivity. Nanotechnology 2014; 25(49): 495602.

33. Wondwossen M, Yadav OP and Tesfahun K. Photo-catalytic removal of methyl orange dye by polyaniline modified $\mathrm{ZnO}$ using visible radiation. STAR J 2014; 3(2): 93-102.

34. Ji Y, Qin C, Niu H, et al. Electrochemical and electrochromic behaviors of polyaniline-graphene oxide composites on the glass substrate/Ag nano-film electrodes prepared by vertical target pulsed laser deposition. Dyes Pigm 2015; 117: 72-82.
35. Zhou C, Li X, Gong X, et al. Ethanol-guided synthesis of (flower-on-leaf)-like aniline oligomers with excellent adsorption properties. New J Chem 2015; 39(12): 9257-9264.

36. Gupta Y, Hellgardt K and Wakeman R. Enhanced permeability of polyaniline based nano-membranes for gas separation. J Membr Sci 2006; 282(1-2): 60-70.

37. Shumakovich G, Streltsov A, Gorshina E, et al. Laccasecatalyzed oxidative polymerization of aniline dimer (N-phenyl-1,4-phenylenediamine) in aqueous micellar solution of sodium dodecylbenzenesulfonate. $\mathrm{J} \mathrm{Mol}$ Catal B: Enzym 2011; 69(3-4): 83-88.

38. Poletto M, Ornaghi $\mathrm{H}$ and Zattera A. Native cellulose: structure, characterization and thermal properties. Materials 2014; 7(9): 6105-6119.

39. Yue L, Zheng Y, Xie Y, et al. Preparation of a carboxymethylated bacterial cellulose/polyaniline composite gel membrane and its characterization. $R S C A d v 2016$; 6(73): 68599-68605.

40. Gupta K, Chakraborty G, Jana PC, et al. Temperature dependent dc and ac electrical transport properties of the composite of a polyaniline nanorod with copper chloride. Solid State Commun 2011; 151(7): 573-578.

41. Xu W, Mu B, Zhang W, et al. Facile fabrication of welldefined polyaniline microtubes derived from natural kapok fibers for supercapacitors with long-term cycling stability. RSC Adv 2016; 6(72): 68302-68311.

42. Nguyen L, Nguyen H, Nguyen N, et al. Portable cholesterol detection with polyaniline-carbon nanotube film based interdigitated electrodes. Adv Nat Sci: Nanosci Nanotechnol 2012; 3(1): 015004.

43. Masibi K, Fayemi O, Adekunle A, et al. Electrocatalysis of lindane using antimony oxide nanoparticles basedSWCNT/PANI nanocomposites. Front Chem 2018; 6: 423.

44. Kizildag N, Ucar N, Oztoksoy ME et al. Poly(vinyl alcohol)/polyaniline (PVA/PANi) conductive nanofibers by electrospinning. In: The International Istanbul Textile Congress 2013, Istanbul, Turkey, 30 May-1 June 2013, pp. 1-6. Istanbul: The International Istanbul Textile Congress.

45. Kim E. Effects of binary doping on chiroptical, electrochemical, and morphological properties of chiral polyaniline. J Korean Chem Soc 2015; 59(5): 423-428.

46. Kakde K. Synthesis and characterization of polyaniline doped with $\mathrm{HCl}, \mathrm{H}_{2} \mathrm{SO}_{4}$ and PVA as secondary dopant for toxic gas (ammonia) sensor. Indian J Sci Technol 2017; 10(20): 1-4.

47. Manjunatha S, Machappa T, Ravikiran Y, Chethan B, Revanasiddappa M. Room temperature humidity sensing performance of polyaniline-holmium oxide composite. Appl Phys A 2019; 125(5).

48. Molina J, Esteves M, Fernández J, Bonastre J, Cases F. Polyaniline coated conducting fabrics: Chemical and electrochemical characterization. Eur Polym J 2011; 47: 2003-2015.

49. Łużny W, Śniechowski M and Laska J. Structural properties of emeraldine base and the role of water contents: X-ray diffraction and computer modelling study. Synth Met 2002; 126(1): 27-35. 
50. Rohom A, Londhe P, Mahapatra S, et al. Electropolymerization of polyaniline thin films. High Perform Polym 2014; 26(6): 641-646.

51. Derun EM, Tugrul N, Senberber FT et al. The optimization of copper sulfate and tincalconite molar ratios on the hydrothermal synthesis of copper borates. Epub ahead of print October 4, 2014. DOI: 10.5281/zenodo.1096563.

52. Vivekanandan J, Ponnusamy V, Mahudeswaran A, et al. Synthesis, characterization and conductivity study of polyaniline prepared by chemical oxidative and electrochemical methods. Arch Appl Sci Res 2011; 3(6): 147-153.
53. Zhang D, Zhang L, Wang B, et al. Nanocomposites of polyaniline and cellulose nanocrystals prepared in lyotropic chiral nematic liquid crystals. J Mater 2013; 614507.

54. Sönmezoğlu S, Taş R, Akın S, et al. Polyaniline microrods based heterojunction solar cell: structural and photovoltaic properties. Appl Phys Lett 2012; 101(25): 253301.

55. Sadi M, Pan J, Xu A, et al. Direct dip-coating of carbon nanotubes onto polydopamine-templated cotton fabrics for wearable applications. Cellulose 2019; 26(12): 7569-7579. 